\title{
HPC clusters and Big Data storage for data analysis in scientific research - Huawei experience
}

\author{
A. Iyudin \\ Huawei Technologies \\ e-mail: iyudin.alexey@huawei.com
}

Key words: high-performance computing, large-scale computing

High-performance computing (HPC for short) is a computer cluster system that connects multiple computer systems using various interconnection technologies and utilizes the integrated computing capability of all connected systems to process large-scale computing tasks. That's why HPC is also known as HPC cluster.

Huawei is dedicated to providing efficient, easy-to-manage, energy-saving, and flexible HPC solutions. Huawei hardware is a cornerstone of the Huawei HPC solution.

Huawei provides a wide range of hardware devices that can be used in the HPC system, including servers, storage devices, and network devices. Huawei servers have bettered 365 world records in SPEC tests, and the number of records is still increasing. Huawei OceanStor 9000 provides up to 5,000,000 operations per second (OPS) and $200 \mathrm{~GB} / \mathrm{s}$ throughput, and features high reliability and optimal performance.

The Huawei HPC solution provides board-level to system-level energy saving measures, intuitive, real-time monitoring, and dynamic energy saving technologies, reducing power consumption by up to $40 \%$.

Huawei has delivered nearly a hundred HPC projects, customers all over the five continents, in education, scientific research, energy, animation, meteorology and other fields to play a role. 